\title{
Preoperative risk factors predict survival following cardiac retransplantation: Analysis of the United Network for Organ Sharing database
}

\author{
Erol Belli, MD, ${ }^{\mathrm{a}}$ Juan Carlos Leoni Moreno, MD, ${ }^{\mathrm{b}}$ Jeffrey Hosenpud, MD,${ }^{\mathrm{b}}$ Bhupendra Rawal, $\mathrm{MS},{ }^{\mathrm{c}}$ and \\ Kevin Landolfo, $\mathrm{MD}^{\mathrm{d}}$
}

Background: The aim of our study was to identify preoperative risk factors affecting overall survival after cardiac retransplantation (ReTX) in a contemporary era.

\begin{abstract}
Methods: The United Network for Organ Sharing database was used to identify patients undergoing ReTX between 1995 and 2012. Of the total 28,464 primary transplants performed, 987 (3.5\%) were retransplants. The primary outcome investigated was overall survival. The influence of preoperative donor and recipient characteristics on survival were then tested with univariate logistic regression and multivariate Cox regression models.
\end{abstract}

Results: Of 987 patients who underwent ReTX, median survival was 9 years. Estimated survival at 1, 3, 5, 10, and 15 years following retransplant was $80 \%$ (95\% confidence interval [CI], $78 \%-83 \%), 70 \%(95 \% \mathrm{CI}$, $67 \%-73 \%$ ), 64\% (95\% CI, 61\%-67\%), 47\% (95\% CI, 43\%-51\%), and 30\% (95\% CI, $25 \%-37 \%$ ), respectively. Clinical predictors of survival using multivariable analysis included donor age (relative risk [RR], 1.14; $P=.004)$, ischemic time $>4$ hours (RR, $1.48 ; P=.004)$; preoperative support with extracorporeal membrane oxygenator (RR, 3.91; $P<.001)$, and the time between previous and current transplant $(P=.004)$. Patients with ReTX have 1.27 times higher relative risk of death compared with patients undergoing primary transplant only (RR, 1.27; 95\% CI, 1.13-1.42; $P<.001$ ).

Conclusions: Patients who undergo cardiac ReTX can expect to have a 1-year survival less than a patient undergoing primary transplant with an acceptable median overall survival. Both donor and recipient preoperative factors contribute to overall survival following cardiac ReTx. Donor characteristics include age of the donor and ischemic time. Recipient factors include the need for extracorporeal membrane oxygenator and the number of days between the first and second transplant. Optimal survival following cardiac ReTX can best be predicted by choosing patients who are farther out from their initial transplant, not dependent upon preoperative extracorporeal support, and by choosing donor hearts younger in age and those likely to have shorter ischemic times. (J Thorac Cardiovasc Surg 2014;147:1972-7)

Supplemental material is available online.

Cardiac retransplantation (ReTX) remains an option for those patients with primary graft failure following

From the Departments of Surgery, ${ }^{\mathrm{a}}$ Cardiology, ${ }^{\mathrm{b}}$ Biostatistics and Bioinformatics, ${ }^{\mathrm{c}}$ and Cardiothoracic Surgery, ${ }^{\mathrm{d}}$ Mayo Clinic Florida, Jacksonville, Fla.

Supported in part by Health Resources and Services Administration contract 234-2005-37011C. The content is the responsibility of the authors alone and does not necessarily reflect the views or policies of the Department of Health and Human Services, nor does mention of trade names, commercial products, or organizations imply endorsement by the US government.

Disclosures: Authors have nothing to disclose with regard to commercial support.

Received for publication Oct 22, 2013; revisions received Jan 20, 2014; accepted for publication Feb 3, 2014; available ahead of print March 15, 2014.

Address for reprints: Kevin Landolfo, MD, 4500 San Pablo Blvd, Jacksonville, FL 32224.

$0022-5223 / \$ 36.00$

Copyright (c) 2014 by The American Association for Thoracic Surgery

http://dx.doi.org/10.1016/j.jtcvs.2014.02.013 transplant. The International Society for Heart and Lung Transplantation (ISHLT) estimates the median survival for primary heart transplants to be 10 years, but little is reported or known about long-term outcomes or survival in recipients of ReTx. ${ }^{1}$ Previous reports have documented 1-year survival between $23 \%$ and $65 \%$ following $\operatorname{ReTX}^{2-7}$ This fact has led some to conclude that ReTX should be limited, given the scarcity of suitable cardiac donors. ${ }^{3-8}$ Our study provides a review of ReTX in the United States from 1995 through 2012 using data submitted to the United Network for Organ Sharing (UNOS) database, a prospectively collected registry that collects voluntary data from all United States transplant centers. This analysis focused on evaluation of overall survival following ReTX and identification of the preoperative donor and recipient risk factors that determine outcomes. This analysis is limited to a contemporary cohort of patients who have undergone cardiac ReTX in the United States from 1995 to 2012 to provide updated information on risk factors and overall survival. 


\section{Abbreviations and Acronyms \\ $\mathrm{ECMO}=$ extracorporeal membrane oxygenator \\ IABP $=$ intra-aortic balloon pump \\ ISHLT $=$ International Society for Heart and Lung Transplantation \\ ReTX = retransplantation \\ UNOS $=$ United Network for Organ Sharing}

\section{METHODS}

\section{Study Patients and Data Collection}

Our review is a retrospective analysis of all 987 patients, at least 18 years of age, from the UNOS database of adult patients who underwent heart ReTX between 1995 and 2010. Information collected regarding recipients (ie, age at initiation, age at retransplant, gender, citizenship, initial body mass index, serum total albumin, ventricular assist device before retransplant, preoperative dependence on inotropes, ventilator status, intra-aortic balloon pump [IABP] status, extracorporeal membrane oxygenation [ECMO] status, diabetes, and dialysis) and information regarding donors (age, gender, and left ventricular ejection fraction of donor hearts). These variables were submitted to univariate analysis. Significant factors were then subjected to multivariate analysis.

\section{Statistical Analysis}

Continuous variables were summarized with the sample median and range. Categorical variables were summarized with number and percentage. The Kaplan-Meier curve analysis along with $95 \%$ confidence intervals (CIs) was used to assess overall survival following ReTX. A single variable Cox proportional hazards regression model was used to evaluate associations of recipient and donor characteristics with overall survival following ReTX; relative risks (RRs) and 95\% CIs were estimated. A multivariable Cox proportional hazards regression model was used to assess if any significant association of recipient and donor characteristics still hold while controlling for other confounding variables. No adjustment for multiple testing was made in these exploratory analyses. Statistical analysis was performed using SAS (version 9.2; SAS Institute, Inc, Cary, NC) and R Statistical Software (version 2.14.0; R Foundation for Statistical Computing, Vienna, Austria).

\section{RESULTS}

A summary of recipient and donor information is displayed in Table 1 for the 987 study recipients. Median age of recipient at retransplant was 50 years (range, 18-74 years). Recipients requiring mechanical circulatory support before ReTX included 38 patients (3.9\%). Most of the recipients were men $(71 \%)$ and US citizens $(99 \%)$. The median initial body mass index of recipients was 25.9 (range, $12.3-50.7)$. The majority of patients $(63 \%)$ had serum total albumin $\leq 3$. Twenty-six recipients $(3 \%)$ were receiving ECMO, $58(6 \%)$ had an IABP, $74(7.5 \%)$ required ventilator support, and a total of $474(48 \%)$ required inotropic support before transplant. Two hundred twenty-four $(23 \%)$ recipients had type 1 or type 2 diabetes diagnosed before transplant and $112(11.5 \%)$ were receiving hemoor peritoneal dialysis before transplant.
The median donor age was 30 years (range, 9-63 years), and $66 \%$ donors were men. Almost all donor hearts $(97 \%)$ had left a ventricular ejection fraction of $50 \%$ or more. The median ischemic time was 3.3 hours (range, 1.0-9.2 hours). The distribution of number of retransplant procedures in the United States from 1995 to 2012 is given in Table 2. The range of number of retransplants was 40 to 71 ; the lowest number of retransplants occurred during 1997 and the highest during 2011. The distribution of retransplants by region is listed in Appendix Table E1.

The median follow-up length was 3.1 years (range, $0-17.1$ years), and a total of 426 patients $(43 \%)$ died during follow-up. Estimated survival at 1, 3, 5, 10, and 15 years following retransplant was $80 \%$ (95\% CI, 78\%-83\%), $70 \%$ (95\% CI, 67\%-73\%), 64\% (95\% CI, 61\%-67\%), $47 \% \quad(95 \% \quad \mathrm{CI}, \quad 43 \%-51 \%)$, and $30 \% \quad(95 \% \quad \mathrm{CI}$, 25\%-37\%), respectively (Table 3 and Figure 1). Associations of survival with recipients and donor characteristics in univariate and multivariate analysis are displayed in Table 4. With the multivariable Cox regression analysis, recipient characteristics associated with increased risk of death included support with ECMO (RR, 3.91; $P<.001$ ), and time between previous transplant and current transplant $(P=.004)$ (Figure 2). Donor characteristics associated with increased risk of death included age $(\mathrm{RR}, 1.14 ; P=.004)$ and ischemic time (RR, 1.48; $P=.004)$. Although univariate analysis demonstrated that the age of recipient at transplant, total serum albumin, need for mechanical ventilation, and presence of IABP had shown significant association with survival, these variables did not meet statistical significance in the multivariate analysis. In comparison with the ReTx patients $>1$ year from primary transplant versus those receiving primary transplants during the same period, ReTX patients have 1.27 times higher RR of death compared with patients with primary transplant only (RR, 1.27; 95\% CI, 1.13-1.42; $P<.001$ ) (Figure 3).

\section{DISCUSSION}

ReTX has remained controversial since the first reports more than 30 years ago. ${ }^{8}$ This was primarily due to the shortage of suitable donors for cardiac transplantation. Currently, 400 patients die every year waiting for a suitable donor heart. The lack of suitable donor hearts, in addition to the poor outcomes historically associated with ReTX, has led several authors to conclude that the practice of cardiac ReTX poses an ethical dilemma and should therefore be limited. ${ }^{9-14}$ Survival following ReTX during the 1970s and the early 1990s was particularly poor in patients receiving retransplant within 1 month of the first transplant. ${ }^{5,6,15}$

Because reports of ReTX emerged from the ISHLT during the early 1990s showing that the 1-year survival following ReTX was only $42 \%$, some members of the European community believed that ReTX should not be 
TABLE 1. Recipient and donor characteristics

\begin{tabular}{|c|c|}
\hline Variable* & $\begin{array}{c}\text { Overall sample } \\
(\mathbf{N}=987) \\
\end{array}$ \\
\hline Age at retransplant (y) & $50(18-74)$ \\
\hline Age at initiation $(\mathrm{y})$ & $49(15-73)$ \\
\hline Sex (male) & 697 (70.6) \\
\hline US citizenship & 973 (99) \\
\hline Initial BMI & $25.9(12.3-50.7)$ \\
\hline \multicolumn{2}{|l|}{ Acute rejection episodes } \\
\hline 1 & $41(7.7)$ \\
\hline 2 & $30(5.6)$ \\
\hline 3 & $464(86.7)$ \\
\hline \multicolumn{2}{|l|}{ Graft failure cause } \\
\hline Hyper acute rejection & $26(35.1)$ \\
\hline Acute rejection & $12(16.2)$ \\
\hline Chronic rejection & $21(28.4)$ \\
\hline Unknown & $15(20.3)$ \\
\hline Days listed as status 1 & $0(0-224)$ \\
\hline Days listed as status 1a & $0(0-692)$ \\
\hline Days listed as status $1 b$ & $0(0-1904)$ \\
\hline Days listed as status 2 & $14(0-2491)$ \\
\hline Ischemic time (h) & $3.3(1.0-9.2)$ \\
\hline Length of hospital stay (d) & $2(0-9)$ \\
\hline \multicolumn{2}{|l|}{ Serum total albumin } \\
\hline$\leq 3$ & $391(63.0)$ \\
\hline$>3$ & $230(37.0)$ \\
\hline VAD before transplant & $38(3.9)$ \\
\hline Cardiac output obtained while on inotropes & $176(22.3)$ \\
\hline Inotropes & $474(48.0)$ \\
\hline Ventilator & $74(7.5)$ \\
\hline IABP & $58(5.9)$ \\
\hline ECMO & $26(2.6)$ \\
\hline \multicolumn{2}{|l|}{ Diabetes } \\
\hline Type 1 & $23(2.4)$ \\
\hline Type 2 & $81(8.3)$ \\
\hline Unknown & $120(12.3)$ \\
\hline \multicolumn{2}{|l|}{ Dialysis at listing } \\
\hline Hemodialysis & $69(7.1)$ \\
\hline Peritoneal dialysis & $4(0.4)$ \\
\hline Unknown & $39(4.0)$ \\
\hline Donor age $(y)$ & $30(9-63)$ \\
\hline Donor sex (male) & $654(66.3)$ \\
\hline \multicolumn{2}{|c|}{ Left ventricular ejection fraction of donor heart $(\%)$} \\
\hline 30 & $4(0.5)$ \\
\hline 40 & $16(2.2)$ \\
\hline$\geq 50$ & $719(97.3)$ \\
\hline
\end{tabular}

The sample median (range) is given for continuous variables and number (percent) is given for categorical variables. $B M I$, Body-mass index; $V A D$, ventricular assist device; $I A B P$, intra-aortic balloon pump; $E C M O$, extracorporeal membrane oxygenation. *Information was not available for the following variables: citizenship $(n=4)$, initial BMI $(n=30)$ acute rejection episodes $(n=452)$, ischemic time $(n=79)$, length of stay $(n=254)$, graft failure cause $(n=913)$, left ventricular ejection fraction of donor heart $(n=248)$, serum total albumin $(n=366)$, cardiac output obtained while on inotropes $(\mathrm{n}=196)$, diabetes $(\mathrm{n}=11)$, and dialysis at listing $(\mathrm{n}=11)$.

performed at all. ${ }^{13}$ A single center from France (Schnetzler and colleagues ${ }^{5}$ ) reported on 24 patients undergoing retransplant compared with 47 patients undergoing primary
TABLE 2. Number of retransplants, by year

\begin{tabular}{lcc}
\hline $\begin{array}{c}\text { Year of } \\
\text { retransplant }\end{array}$ & $\begin{array}{c}\text { Retransplant } \\
\text { sample (N = 987) }\end{array}$ & $\begin{array}{c}\text { Proportion of total heart } \\
\text { transplants in United States }\end{array}$ \\
\hline 1995 & $47(4.8)$ & $47 / 2363(2.0)$ \\
1996 & $44(4.5)$ & $44 / 2343(1.9)$ \\
1997 & $40(4.1)$ & $40 / 2293(1.7)$ \\
1998 & $67(6.8)$ & $67 / 2348(2.9)$ \\
1999 & $55(5.6)$ & $55 / 2188(2.5)$ \\
2000 & $42(4.3)$ & $42 / 2199(1.9)$ \\
2001 & $49(5.0)$ & $49 / 2202(2.2)$ \\
2002 & $51(5.2)$ & $51 / 2155(2.4)$ \\
2003 & $42(4.3)$ & $42 / 2057(2.0)$ \\
2004 & $51(5.2)$ & $51 / 2015(2.5)$ \\
2005 & $64(6.5)$ & $64 / 2125(3.0)$ \\
2006 & $53(5.4)$ & $53 / 2193(2.4)$ \\
2007 & $60(6.1)$ & $60 / 2209(2.7)$ \\
2008 & $64(6.5)$ & $64 / 2163(3.0)$ \\
2009 & $60(6.1)$ & $60 / 2211(2.7)$ \\
2010 & $67(6.8)$ & $67 / 2332(2.9)$ \\
2011 & $71(7.2)$ & $71 / 2322(3.1)$ \\
2012 & $60(6.1)$ & $60 / 2378(2.5)$ \\
\hline
\end{tabular}

Values are given as $\mathrm{n}(\%)$.

transplant as controls. Survival at 1 and 5 years for ReTX were $45.5 \%$ and $31.2 \%$, respectively, compared with $59.4 \%$ and $38.8 \%$ for controls. ${ }^{5}$ In another report ${ }^{16}$ comparing the survival of patients undergoing retransplant at a single US institution compared with data from the ISHLT, survival was found to be as high as $64 \%$ at 1 year, provided that certain criteria were met. They identified the "ideal candidate" for ReTX as a patient who had an interval between transplants $>6$ months, a date of second operation after 1985, and without preoperative mechanical support. ${ }^{16}$ Despite these attempts to refine the criteria for ReTX, many centers still refused to adopt this practice.

TABLE 3. Survival following retransplant procedure

\begin{tabular}{|c|c|}
\hline $\begin{array}{l}\text { Length of time after } \\
\text { retransplant }(y)\end{array}$ & Proportion of survival $(95 \% \mathrm{CI})$ \\
\hline 1 & $80(78-83)$ \\
\hline 2 & 75 (72-78) \\
\hline 3 & $70(67-73)$ \\
\hline 4 & $68(65-71)$ \\
\hline 5 & $64(61-67)$ \\
\hline 6 & $60(57-64)$ \\
\hline 7 & $56(53-60)$ \\
\hline 8 & $54(50-58)$ \\
\hline 9 & $50(46-54)$ \\
\hline 10 & $47(43-51)$ \\
\hline 11 & $44(40-48)$ \\
\hline 12 & $41(37-46)$ \\
\hline 13 & $38(33-43)$ \\
\hline 14 & $36(31-41)$ \\
\hline 15 & $30(25-37)$ \\
\hline
\end{tabular}

Values are given as \%. CI, Confidence interval. 


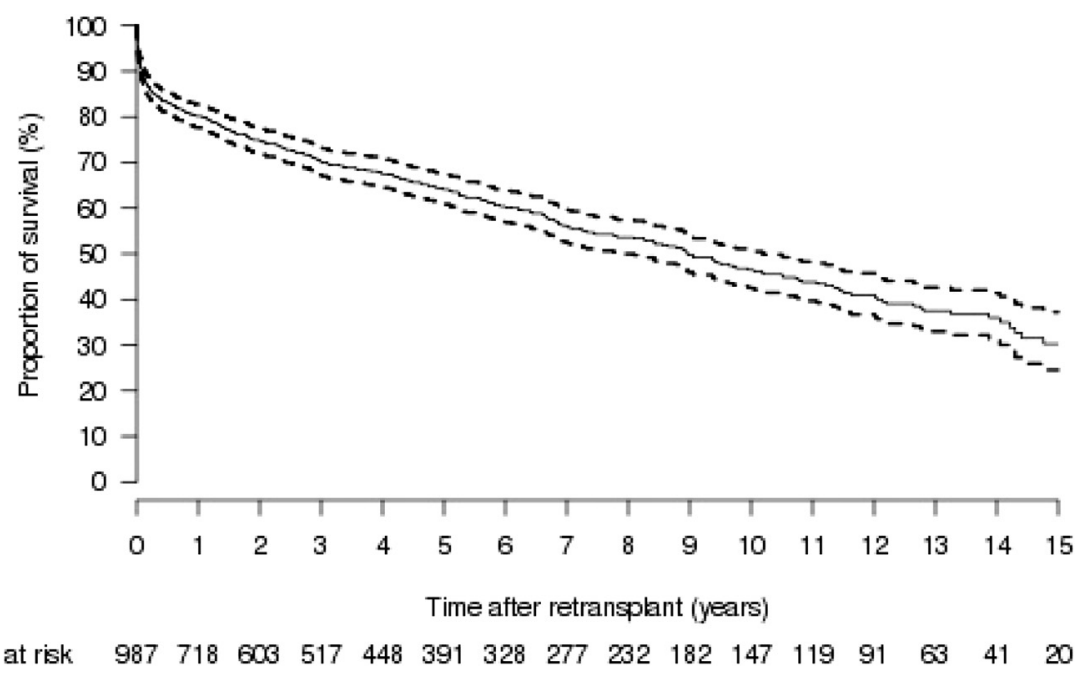

FIGURE 1. Survival following retransplantation. Dashed lines represent $95 \%$ confidence intervals.

More recent data have shown improved survival following ReTX. Srivastava and colleagues, ${ }^{6}$ using ISHLT registry data from 1987 through 1998, reported a 65\% 1 -year survival for 514 patients undergoing ReTX. Another important conclusion from that study was that overall survival following ReTX approached that of primary transplant if the interval between transplants was $>2$ years. These conclusions, however, were only valid for centers performing at least 9 heart transplants per year and for patients who were not dependant on a ventilator or a left ventricular assist device.

The data presented in our study are unique in that the population studied represents a large sample of patients undergoing ReTX subject to contemporary selection processes, surgical techniques, and methodology that therefore provides a unique perspective on patients undergoing ReTX. In our large cohort, survival at 1,3 , and 5 years was $80 \%$, $70 \%$, and $64 \%$, respectively, which is significantly higher than that previously published. The reasons for improved survival have not yet been clearly elucidated; however, more refined retransplant candidate screening selection processes; improvement in surgical and perioperative techniques; and better posttransplant medical protocols, including advances in immunosuppressive therapy, all serve as potential explanations.

Previously published studies have shown that preoperative mechanical ventilation, high serum creatinine, and older recipient age are associated with poorer graft

TABLE 4. Associations of recipient and donor characteristics with overall survival following retransplant*

\begin{tabular}{|c|c|c|c|c|}
\hline \multirow[b]{2}{*}{ Variable } & \multicolumn{2}{|c|}{ Single variable analysis } & \multicolumn{2}{|c|}{ Multivariable analysis } \\
\hline & RR $(95 \%$ CI $)$ & $P$ value & $\mathbf{R R}(95 \% \mathbf{C I})$ & $P$ value \\
\hline Age at retransplant (10-y increase) & $1.08(1.01-1.15)$ & .034 & $1.08(0.99-1.17)$ & .072 \\
\hline Gender (male) & $0.85(0.69-1.04)$ & .12 & NI & N/A \\
\hline Donor age (10-y increase) & $1.11(1.03-1.20)$ & .004 & $1.14(1.05-1.25)$ & .004 \\
\hline \multicolumn{5}{|c|}{ Time between previous transplant and retransplant $(\mathrm{y}) \dagger$} \\
\hline$\leq 1$ & 1.00 (reference) & N/A & 1.00 (reference) & N/A \\
\hline$>1 \leq 5$ & $0.54(0.38-0.75)$ & $<.001$ & $0.66(0.44-1.01)$ & .053 \\
\hline$>5$ & $0.43(0.32-0.58)$ & $<.001$ & $0.53(0.36-0.78)$ & .001 \\
\hline Ischemic time $(>4 \mathrm{~h})$ & $1.52(1.20-1.92)$ & $<.001$ & $1.48(1.12-1.87)$ & .004 \\
\hline Serum total albumin $(>3)$ & $0.71(0.54-0.94)$ & .017 & NI & N/A \\
\hline Inotropes & $1.14(0.95-1.38)$ & .17 & NI & N/A \\
\hline Ventilator & $2.44(1.81-3.29)$ & $<.001$ & $1.29(0.82-2.03)$ & .27 \\
\hline IABP & $1.86(1.32-2.63)$ & $<.001$ & $1.03(0.66-1.60)$ & .90 \\
\hline ECMO & $5.68(3.60-8.97)$ & $<.001$ & $3.91(2.08-7.38)$ & $<.001$ \\
\hline Diabetes & $1.21(0.97-1.51)$ & .092 & NI & N/A \\
\hline Dialysis at listing & $1.43(1.09-1.90)$ & .011 & $1.12(0.77-1.63)$ & .56 \\
\hline
\end{tabular}

$R R$, Relative risk; $C I$, confidence interval; $N I$, not included in the multivariable analysis; $N / A$, not applicable; $I A B P$, intra-aortic balloon pump; $E C M O$, extracorporeal membrane oxygenation. *RRs, $95 \%$ CIs, and $P$ values result from Cox proportional hazards regression models. Relative risks correspond to presence of the given characteristic (categorical variables) or the increase given in parenthesis (continuous variables). All variables with $P$ value $\leq .05$ in a single variable analysis were included in multivariable analysis $(\mathrm{n}=797)$, except serum total albumin because of the large amount of missing information (missing $=366)$ with this variable. $\dagger$ Test of overall difference for single-variable analysis: $P<.001$. Test of overall difference for multivariable analysis: $P=.003$. 


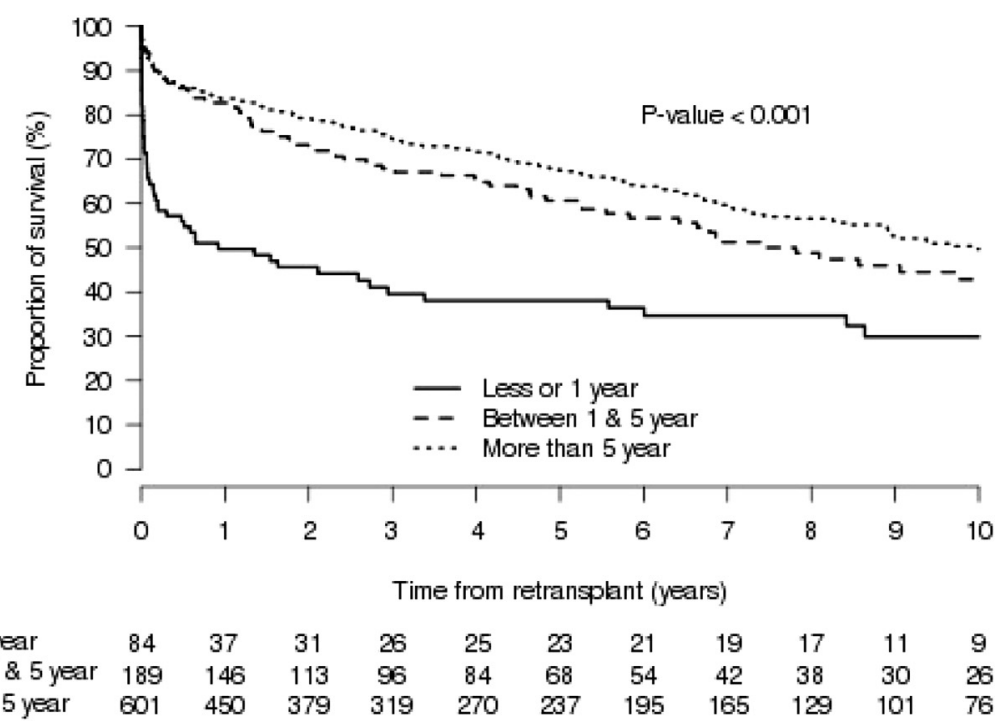

FIGURE 2. Survival following retransplantation according to the time between previous transplant and retransplant.

survival. ${ }^{17}$ The data from our study emphasize both donor and recipient risk factors. Univariate analysis showed that donor age; recipient age; days between first transplant and ReTX; serum albumin; ventilator status; and pretransplant dependence on IABP, ECMO, or dialysis were significant predictors of outcome.

Regarding recipient risk factors for decreased survival following ReTX, further stratification with multivariate analysis demonstrated reduced survival for recipients requiring pretransplant ECMO and those having had a shorter time between transplants. Patients who received an immediate transplant for primary graft failure had a much worse prognosis than those who had an indolent course of chronic heart failure with a longer time course after their initial transplant. Although current guidelines from the American Society of Transplant Surgeons suggest the interval between transplants be at least 6 months, ${ }^{3}$ data from our study suggest that optimal survival following ReTX occurs when the interval between the initial transplant and retransplant is at least 1 year, with at least 5 years being the optimal interval. According to the data reported in our study, dependence on ECMO before transplant confers a dismal prognosis and therefore should serve as a relative contraindication to ReTX. Mechanical support with ECMO was the strongest RR factor for decreased survival. Contrary to previously published reports, recipient factors not found to be statistically significant included pretransplant ventilator status and dependence on inotropic support. The majority of patients undergoing ReTX were, in fact, treated with inotropes without a significant effect on overall patient survival following transplant.

Donor factors significant for reduced survival after ReTX by multivariate analysis included the donor heart age and total ischemic time after harvest.

In the current era, the annual number of ReTX procedures reported in the United States consistently represents $2 \%$ to $3 \%$ of all transplants. These data were obtained from the UNOS database, which is not an audited registry. Because these data are obtained and reported voluntarily, all outcomes data regarding patients undergoing ReTX may not

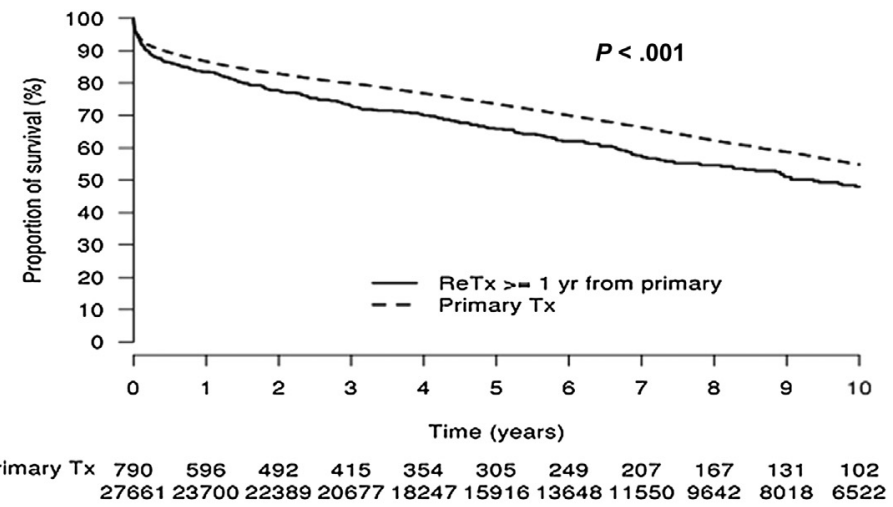

FIGURE 3. Survival following retransplantation $(R e T X)>1$ year from primary transplant $(T X)$ versus primary TX. 
have been captured, which serves as an important limitation of our study.

Despite this limitation, our study does allow us to draw several important conclusions regarding improved survival following ReTX that contradicts historical data and published guidelines. Most notably, patients who have survived at least 1 year from the primary transplant have better overall survival compared with those who are $<1$ year out from their initial surgery. Patients surviving at least 5 years from their initial transplant had optimal survival following ReTX. The reason for the improved survival observed following ReTX in our study is likely multifactorial. The reasons for this positive change may be related to improvements in modern therapy, improved immunosuppressive regimens, and/or better selection of retransplant candidates. Nevertheless, patients undergoing ReTX even 1 year from primary transplant continue to have a lower overall survival compared with primary transplants in the same era. The difference may be less than anticipated by transplant centers.

\section{CONCLUSIONS}

ReTX remains a viable option for a limited transplant population. Optimal candidates for consideration of ReTX include younger recipients who are at least 1 year out from their primary transplant. ECMO before ReTX is the strongest negative predictor of overall survival and should therefore be considered a relative contraindication to ReTX. Inotropic support before ReTX has no effect on overall survival and should not preclude a patient from ReTX. Cardiac ReTX continues to grow as a viable option for transplant recipients with primary graft failure.

\section{References}

1. Stehlik J, Edwards LB, Kucheryavaya AY, Aurora P, Christie JD, Kirk R, et al. The Registry of the International Society for Heart and Lung Transplantation: twenty-seventh official adult heart transplant report-2010. J Heart Lung Trans plant. 2010;29:1089-103.

2. Chou NK, Chi NH, Chen YS, Yu HY, Lee CM, Huang SC, et al. Heart retransplantation for heart allograft failure in Chinese heart transplant recipients: NTUH experience. Transplant Proc. 2006;38:2147-8.

3. Johnson MR, Aaronson KD, Canter CE, Kirklin JK, Mancini DM, Mehra MR, et al. Heart retransplantation. Am J Transplant. 2007;7:2075-81.

4. Schlechta B, Kocher AA, Ehrlich M, Ankersmit J, Ploner M, Walch K, et al Heart retransplantation: institutional results of a series of 31 cases. Transplan Proc. 2001;33:2759-61.

5. Schnetzler B, Pavie A, Dorent R, Camproux AC, Leger P, Delcourt A, et al. Heart retransplantation: a 23-year single-center clinical experience. Ann Thorac Surg. 1998;65:978-83.

6. Srivastava R, Keck BM, Bennett LE, Hosenpud JD. The results of cardiac retransplantation: an analysis of the Joint International Society for Heart and Lung Transplantation/United Network for Organ Sharing Thoracic Registry Transplantation. 2000;70:606-12.

7. Vistarini N, Pellegrini C, Aiello M, Alloni A, Monterosso C, Cattadori B, et al. Should we perform heart retransplantation in early graft failure? Transpl Int. 2010;23:47-53.

8. Copeland JG, Griepp RB, Bieber CP, Billingham M, Schroeder JS, Hunt S, et al. Successful retransplantation of the human heart. J Thorac Cardiovasc Surg. 1977;73:242-7.

9. Collins EG, Mozdzierz GJ. Cardiac retransplantation: determining limits. Heart Lung. 1993;22:206-12.

10. Evans RW, Manninen DL, Dong FB, McLynne DA. Is retransplantation cost effective? Transplant Proc. 1993;25:1694-6.

11. Meerdink P. Heart retransplantation: what is the right choice? Prog Cardiovasc Nurs. 2001;16:39-40.

12. Meiser BM, Uberfuhr P, Stang A, Ingrisch S, Wenke K, Kreuzer E, et al Retransplantation of an already transplanted heart. Transplant Proc. 1992; 24:2663-4.

13. Mullins P, Scott J, Chauhan A, Graham T, Aravot D, Large S, et al. Acute heart retransplantation. Lancet. 1991;337:1552-3.

14. Novick RJ. Heart and lung retransplantation: should it be done? J Heart Lung Transplant. 1998; 17:635-42.

15. Smith JA, Ribakove GH, Hunt SA, Miller J, Stinson EB, Oyer PE, et al. Heart retransplantation: the 25-year experience at a single institution. J Heart Lung Transplant. 1995; 14:832-9.

16. Karwande SV, Ensley RD, Renlund DG, Gay WA Jr, Richenbacher WE Doty DB, et al. Cardiac retransplantation: a viable option? The Registry of the International Society for Heart and Lung Transplantation. Ann Thorac Surg. 1992:54:840-4.

17. Kilic A, Weiss ES, Arnaoutakis GJ, George TJ, Conte JV, Shah AS, et al. Identifying recipients at high risk for graft failure after heart retransplantation. Ann Thorac Surg. 2012;93:712-6. 
APPENDIX TABLE E1. Distribution of patients by US regions and US states and territories

Region, state,* or territory

Overall sample $(\mathrm{N}=\mathbf{9 8 7})$

US region

1
2
3
4
5
6
7
8
9
10
11

US state or territory

Alaska

Alabama

Arkansas

Arizona

California

Colorado

Connecticut

Washington, DC

Delaware

Florida

Georgia

Hawaii

Iowa

Idaho

Illinois

Indiana

Kansas

Kentucky

Louisiana

Massachusetts

Maryland

Maine

Michigan

Minnesota

Missouri

Mississippi

Montana

North Carolina

North Dakota

Nebraska

New Hampshire

New Jersey

New Mexico

Nevada

New York

Ohio

Oklahoma

Oregon

$29(2.9)$

131 (13.3)

$136(13.8)$

$93(9.4)$

233 (23.6)

25 (2.5)

$70(7.1)$

$70(7.1)$

$76(7.7)$

$69(7.0)$

55 (5.6)

$1(0.1)$

13 (1.3)

15 (1.5)

$13(1.3)$

187 (19.1)

29 (3)

10 (1)

$1(0.1)$

$5(0.5)$

$61(6.2)$

25 (2.6)

$1(0.1)$

$7(0.7)$

$6(0.6)$

$28(2.9)$

28 (2.9)

$13(1.3)$

$7(0.7)$

16 (1.6)

11 (1.1)

$22(2.3)$

$2(0.2)$

$18(1.8)$

$16(1.6)$

$13(1.3)$

$9(0.9)$

$4(0.4)$

27 (2.8)

$1(0.1)$

$6(0.6)$

$3(0.3)$

$56(5.7)$

$3(0.3)$

$2(0.2)$

$58(5.9)$

$24(2.5)$

$6(0.6)$

$9(0.9)$

$48(4.9)$

$6(0.6)$

$3(0.3)$
APPENDIX TABLE E1. Continued

Region, state,* or territory

Overall sample $(\mathbf{N}=\mathbf{9 8 7})$

South Carolina

10 (1)

South Dakota

$1(0.1)$

Tennessee

$15(1.5)$

Texas

$81(8.3)$

Utah

$12(1.2)$

Virginia

$6(0.6)$

Vermont

$2(0.2)$

Washington

$13(1.3)$

Wisconsin

$21(2.2)$

West Virgina

$2(0.2)$

Wyoming

$1(0.1)$

Values are given as $\mathrm{n}(\%) . *$ Missing information: States $(\mathrm{n}=10)$. 